This copy is for your personal, non-commercial use only.

If you wish to distribute this article to others, you can order high-quality copies for your colleagues, clients, or customers by clicking here.

Permission to republish or repurpose articles or portions of articles can be obtained by following the guidelines here.

The following resources related to this article are available online at www.sciencemag.org (this information is current as of September 4, 2012 ):

Updated information and services, including high-resolution figures, can be found in the online version of this article at:

http://www.sciencemag.org/content/333/6039/182.full.html

A list of selected additional articles on the Science Web sites related to this article can be found at:

http://www.sciencemag.org/content/333/6039/182.full.html\#related

This article has been cited by 1 articles hosted by HighWire Press; see:

http://www.sciencemag.org/content/333/6039/182.full.html\#related-urls

This article appears in the following subject collections:

Astronomy

http://www.sciencemag.org/cgi/collection/astronomy 
REVIEW

\section{The Coevolution of Galaxies and Supermassive Black Holes: A Local Perspective}

\author{
Timothy M. Heckman ${ }^{1_{\star}}$ and Guinevere Kauffmann ${ }^{2}$
}

One of the most fascinating discoveries in the past decade was that galaxies typically contain a centrally located black hole with a mass that is millions or even billions of times that of the Sun. There is now compelling evidence that we cannot understand how galaxies formed and evolved without understanding the life cycles of these supermassive black holes (and vice versa). We summarize the current understanding of this coevolution of galaxies and supermassive black holes (based largely on observations of the local, present-day universe) and describe prospects for the future.

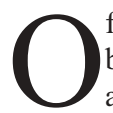
$f$ all the denizens of the cosmos studied by astronomers, none are as fascinating as black holes: objects whose gravitational field is so strong that light itself cannot escape from inside their aptly named "event horizon." Predicted long ago, the existence of these exotic objects has now been established observationally. Black holes come in two varieties: The first have masses roughly 10 times that of our Sun and are formed during the cataclysmic events at the death of a very massive star. The second are found in the centers ("nuclei") of galaxies and have masses that range roughly between one million and one billion times the Sun's mass. For this reason, they are dubbed supermassive black holes (1).

One of the most unexpected and important discoveries of the past decade has been that the lives of galaxies and their supermassive black holes are inextricably intertwined. This is noteworthy, because the scales of the two phenomena are so different: The galaxy is more than one billion times larger than the black hole and contains more than one thousand times as much mass. Many astronomers now believe that we cannot understand how galaxies formed and evolved without understanding their black holes in detail. Understanding the link between galaxies and their black holes has become one of the most important problems in astrophysics.

\section{Background}

The mass of an object can be determined by its gravitational effect on satellite systems orbiting about the object: Measure the speed of the satellite and the radius of its orbit to determine the mass of the central object. Such measurements have revealed the presence of a very large but invisible

${ }^{1}$ Center for Astrophysical Sciences, Department of Physics and Astronomy, Johns Hopkins University, Baltimore, MD 21218, USA. ${ }^{2}$ Max Planck Institute for Astrophysics, Garching, Germany.

*To whom correspondence should be addressed. E-mail: heckman@pha.jhu.edu

an immense amount of radiation. Once beyond the event horizon, this gas can no longer be seen. However, it does add to the mass of the black hole. This is a major way by which black holes grow, but as we will discuss, in some feeding frenzies black holes may also gain mass when they swallow each other!

In addition to the radiation emitted by in-falling hot gas, supermassive black holes can produce powerful "jets." These jets are two very narrow streams of material traveling in opposite directions outward from the black hole at velocities close to the speed of light. The particles in the jet travel out to distances far larger than the galaxy, where they finally decelerate as they interact with surrounding gas and light up in two radio-emitting lobes.

Einstein's theory of relativity tells us that the maximum amount of energy $E$ that can be extracted from a mass $m$ is $E=m c^{2}$ (the complete conversion of mass into energy) ( $c$, the speed of light in a vac-

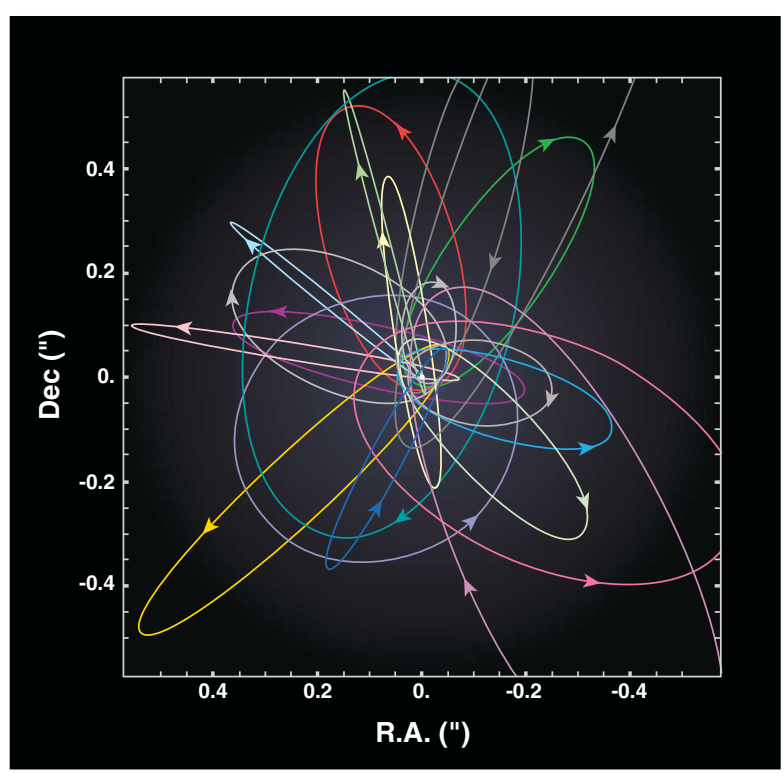

Fig. 1. Plot of the orbits of stars around the supermassive black hole in the nucleus of our own Milky Way. The supermassive black hole lies at the origin of the coordinate system, and the region shown is $\sim 0.15$ light years across. Applying Newton's law of gravity to these orbits yields a black hole mass 4 million times that of the Sun. Dec (declination) and R.A. (right ascension) are the sky coordinates relative to the supermassive black hole measured in seconds of arc ("). [Source: S. Gillesen et al., Max Planck Institute for Extraterrestrial Physics]

mass concentration in the centers of 50 nearby galaxies (1). In our own Milky Way (Fig. 1), it is established that the mass is concentrated within such a small radius that ordinary matter would have no other option but to collapse into the event horizon of a supermassive black hole $(2,3)$.

Today, most supermassive black holes are in a relatively dormant state, producing little energy (4). However, these black holes do experience occasional episodes of intense growth ("feeding frenzies") when they swallow nearby gas clouds. As this gas swirls around the black hole onto the event horizon, it is heated to high temperatures and emits uum). Accretion of matter by a black hole releases roughly $10 \%$ of $m c^{2}$, whereas nuclear reactions in stars extract only $0.7 \%$. This high efficiency means that supermassive black holes in a feeding frenzy are the most powerful known sources of energy: They can literally outshine the entire galaxy in which they live. They are cosmic beacons visible clear across the universe. By measuring their numbers and their properties, we can trace the growth of supermassive black holes across the whole sweep of cosmic time $(5,6)$.

A galaxy such as our Milky Way contains ordinary matter in the form of stars and gas. The stars are arrayed in two primary components: (i) a roughly spherical and slowly rotating "bulge," which dominates the inner part of the galaxy, and (ii) a very flat and more rapidly rotating "disk" that dominates over the bulge at larger radii (Fig. 2). These two components make up the classical visible region of a galaxy, which lies at the center of a much larger halo. The halo consists primarily of dark matter, but also contains a major reservoir of gas that will accrete onto the galaxy and provide the raw material to form new stars and grow the black hole.

Galaxies can be divided into two main populations $(7,8)$. One population consists of galaxies that have young, hot stars that are currently forming out of cold, dense gas. These are the "living" galaxies. The other class consists of galaxies with low star-formation rates and gas fractions. These are the "passive" galaxies. Observations show that the fraction of passive galaxies has increased substantially over the past 8 billion years $(9,10)$, presumably 


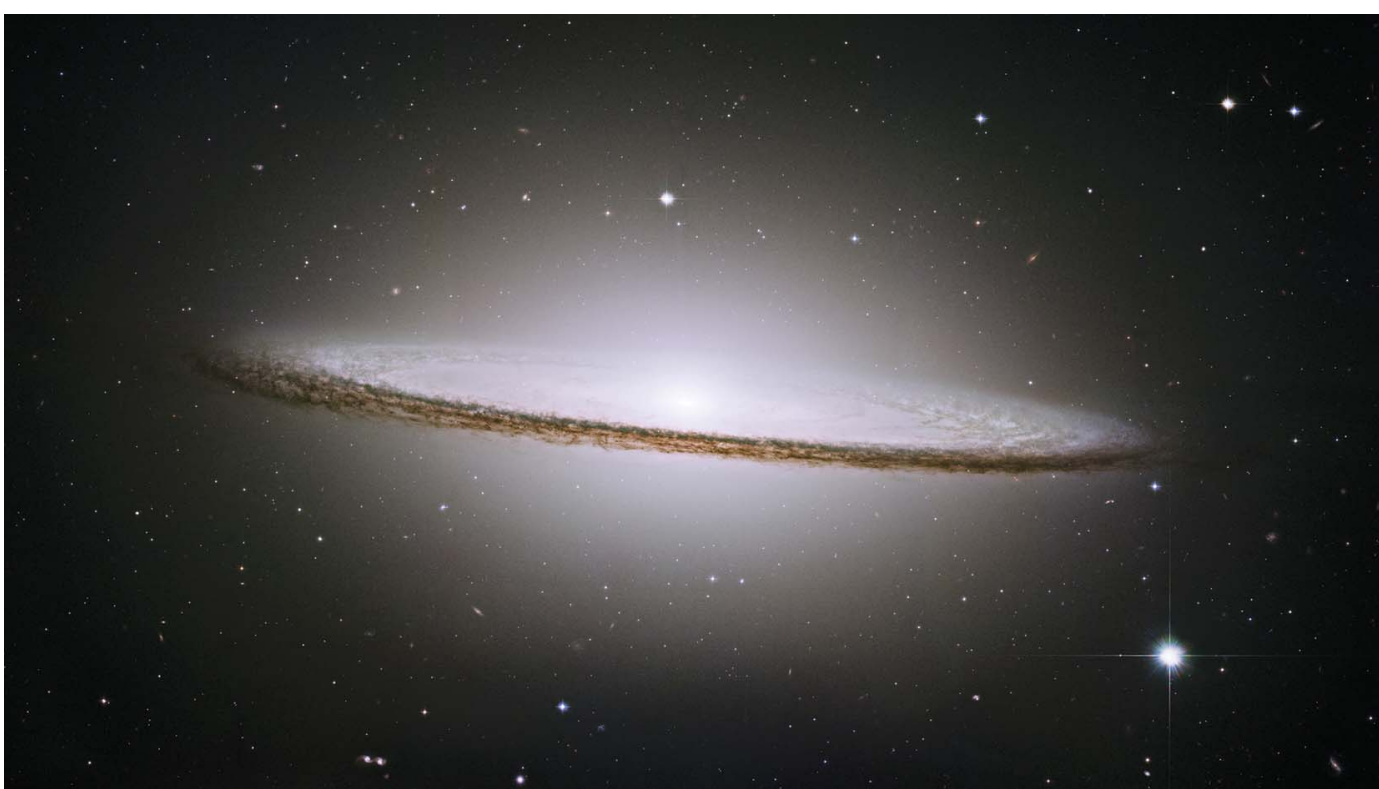

Fig. 2. Hubble Space Telescope image of the Sombrero Galaxy. There are two components to spiral galaxies like this one: (i) a roughly spherical bulge whose light dominates the inner region of the galaxy, and (ii) a thin disk component that dominates the light in the outer region. This disk component is seen nearly edge-on in the Sombrero Galaxy. The disk contains opaque clouds of gas and dust that appear in this image as a black band of obscuration. [Source: NASA/European Space Agency (ESA)/Space Telescope Science Institute (STSCl)/Association of Universities for Research in Astronomy (AURA)/The Hubble Heritage Team] fuels an intense burst of star formation and delivers a fresh supply of gas to the supermassive black hole. The scrambled disk material settles into a newly created bulge. If the merging galaxies contained their own supermassive black holes, these too would merge to form a single larger one.

The release of energy from the merger-induced starburst is so intense that it may blow away most or all of the remaining gas in a powerful outflow. In addition, jets from the black hole may pump energy into the surrounding gas, preventing it from cooling, falling into the galaxy, and forming new stars. The end result of this process is a single galaxy with a larger bulge than was present in either progenitor galaxy, as well as a substantially more massive black hole. In some cases, star formation will cease, causing a living galaxy to be converted into a passive one. Because jets release their energy most efficiently in the hot gaseous material that sur-

because some process has disrupted the cold gas supply in many members of the living population.

\section{Coevolution: The Evidence}

There are two main lines of evidence that the life cycles of galaxies and supermassive black holes are strongly linked. With the help of the Hubble Space Telescope as well as large ground-based observatories, astronomers were able to assemble a sample of supermassive black holes with accurate mass determinations $(1,11)$. To their surprise, the astronomers then found that the mass of each supermassive black hole was roughly a fixed fraction (about one part in one thousand) of the mass of its bulge $(1,11-13)$. This discovery implied that processes acting over billions of years of cosmic time in galaxies must have caused 0.1 percent of the mass of stars forming the bulge to be fed into the black hole. Because bulge formation is believed to be a highly complex process, this is a rather surprising conclusion.

A second, complementary line of evidence comes from using telescopes as time machines to probe the past histories of galaxies. Because of the finite velocity of light, the deeper out we probe in distance, the farther we look back in time. We have now managed to detect galaxies and supermassive black holes at a time when the universe was less than one billion years old. When we track the growth rates of galaxies and supermassive black holes as a function of cosmic time, we see very similar trends for both populations ( 6 ). In particular, the cosmic histories of the growth rates of both galaxies and supermassive black holes depend very strongly on mass $(5,14,15)$. The most massive galaxies and black holes grew rapidly early in the history of the universe, and their masses then remained nearly constant. In contrast, lower-mass galaxies and black holes have grown at a more uniform rate over the entire history of the universe. Put another way, the sites of the most vigorous starformation and the most active black hole growth have moved from the most massive galaxies to progressively smaller and smaller systems as the universe evolved (16-18).

Given the marked similarity in the histories of galaxy and black hole growth, the natural conclusion is that the formation and evolution of these two kinds of objects are tightly linked. The important question is how we can explain this in physical rather than phenomenological terms.

\section{The Current Theoretical Paradigm}

Theoretical models for the coevolution of galaxies and supermassive black holes are based on combining analytic models or numerical simulations of structure formation in the dark matter component of the universe, with educated guesses about how small-scale processes such as star formation and black hole accretion operate in practice (19-22).

Over the course of cosmic time, galaxies grow through two main mechanisms: accretion of gas from the external environment and mergers between two or more galaxies. In galaxy mergers, the thin and highly ordered disk component of each galaxy is scrambled. During the merger, tidal forces between the two galaxies drain away angular momentum from the cold gas in the disk of the galaxy, allowing it to flow into the inner region, where it rounds galaxies located in massive dark-matter halos, death comes earliest to galaxies in the crowded environments within such halos.

\section{Testing the Paradigm with Present-Day Galaxies}

This paradigm does a reasonably good job of reproducing the global statistical properties of the present-day galaxy population, such as the relative numbers of galaxies with different stellar masses and star-formation rates. However, this does not prove that the assumptions incorporated in the model are correct. To test this, we need to demonstrate that the same model can reproduce a wide variety of observational data, not only for presentday galaxies and supermassive black holes, but also in the early universe.

Much more detailed information is currently available for present-day galaxies, because the objects are nearer and easier to study. We will therefore emphasize observational tests in the presentday universe in this article. However, we will also comment on future prospects for investigating the early (distant) universe when the most massive galaxies and black holes were rapidly growing.

The most massive galaxies and their black holes. The most massive black holes today (masses of about 100 million to a few billion times the mass of the Sun) are no longer accreting a substantial amount of gas; thus, their masses are growing very slowly $(14,15)$. These black holes are found in the most massive galaxies with the most massive bulges $(12,13)$. Such galaxies are currently forming stars at a much smaller rate than in the distant past, and are usually lacking a 
major supply of the cold, dense gas needed to form new stars $(8,23)$.

Studies of massive galaxies with X-ray observatories in space indicate that the majority are surrounded by a halo of hot and tenuous gas (24). Black holes in these galaxies are observed to produce large-scale jets. The jets emit electromagnetic radiation that is most easily observed using radio telescopes. The combination of radio and x-ray observations of nearby groups and clusters led to the discovery that jets are able to dump considerable energy into the hot gas atmospheres surrounding nearby massive galaxies (Fig. 3). If cold, dense gas is present it too can be heated by the jet. The heating of gas may be sufficient to prevent the gas from cooling and forming stars (24-26). These observations provided the main motivation for including feedback from jets in the theoretical models.

Intermediate mass galaxies and black holes. Less massive black holes in the present universe (masses of roughly 1 million to 100 million times the mass of the Sun) are still accreting gas at a substantial rate, on average $(14,15)$. However, only a small fraction of these black holes are accreting at very high rates at any given time. This means that the growth occurs sporadically, presumably when gas is able to refuel the accretion disk surrounding the black hole. When activated, these black holes produce a copious supply of light, but they do not produce large and powerful jets (24).

There is a strong connection observed between the growth of these black holes and the formation of stars in the bulgedominated inner region of the galaxy (27-30). If we average over a suitably large sample of such black holes, we find that the ratio between the rate of starformation in the inner regions of the galaxy and the rate of black hole growth is about 1000 (14).

One thousand-to-one is the same as the ratio of the mass of stars to that in the black hole for the most massive galaxies. As we have discussed, stars and black holes in such systems were formed at very early epochs. Thus, the process that determined the 1000-to- 1 ratio in the early universe is still in action today. What exactly is this process? If we knew, it would be very good news for observers, because they would then be able to study its inner workings in a tremendous amount of detail, using observational facilities on the ground and in space. For the moment, all we have are some educated guesses.

\section{Testing Model Assumptions}

Fueling. The hypothesis made in many theoretical models is that supermassive black holes experience major growth episodes during a merger between two galaxies, because this process delivers a fresh supply of fuel to the black hole. Observations show that there is a very high concentration of gas and a high star-formation rate in the central regions of

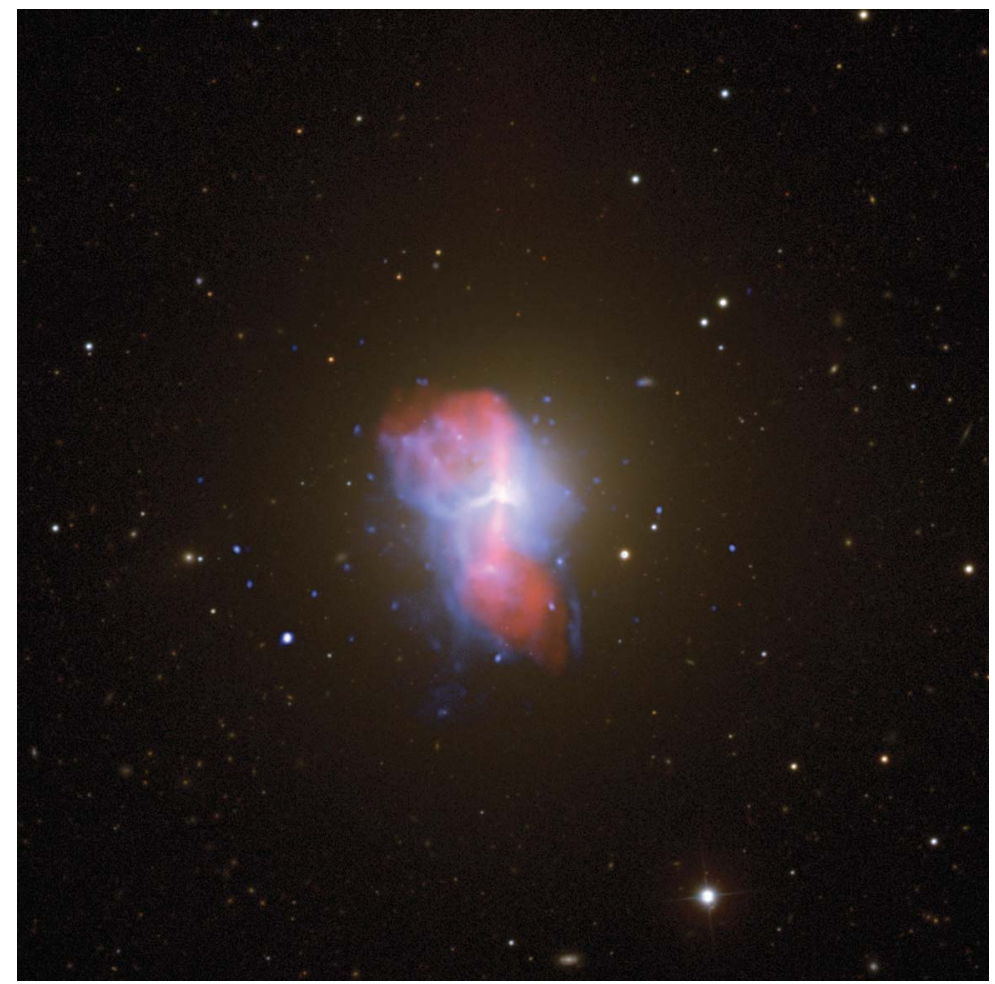

Fig. 3. Twin jets produced by the supermassive black hole in the galaxy Messier 84 are strongly interacting with the hot tenuous gas in the galaxy's halo. The black hole is located at the center of the brightest region in this image. The jets are traced by their gas is traced by its X-ray emission (shown in blue), as mapped with the Chandra X-ray Observatory. The jets decellerate as they travel out into the halo and light up as two dio emission. These lobes form two cavities in the hot gas as they push it out of their way. [Source: $x$-ray, NASA/Chandra X-ray Center (CXC)/Max Planck Institute for Extraterrestrial Physics/A. Finoguenov; radio, NSF/National Radio Astronomy Observatory/NLA/European Southern Observatory/R. A. Laing; optical, Sloan Digital Sky Survey]

and the growth of a supermassive black hole, suggesting a common source of fuel. Star formation equires a supply of cold dense gas, but a merger as is transported to the central region of the galall the way inward to a region that is millions of times smaller, where it can be ingested by the supermassive black hole. How this happens is still a mystery. In particular, we do not know why the ratio of the amount of star formation and black hole growth is 1000 in bulges.

Feedback. If large-scale dynamical processes such as mergers do not regulate the growth of supermassive black holes, some other mechanism must be found to explain the fixed 1000to- 1 ratio between bulge mass and black hole mass. The coevolution of galaxies and supermassive black holes clearly requires more than a common source of fuel. It also requires some physical communication between the black hole and galaxy that limits the growth of each component-in other words, some type of feedback.

Two modes of feedback are well established observationally. In the case of the most massive black holes, feedback is observed to be provided by jets (Fig. 3). Upon reaching the galaxy's halo, the jets deposit energy that displaces and heats the halo gas. This process may limit the growth of the most massive galaxies and supermassive black holes by inhibiting the ability of the hot gas to cool and form stars (24-26).

In lower-mass galaxies, the rapid growth of black holes is associated with high rates of star formation that produce many young stars that emit copious amounts of ultraviolet radiation.

merging galaxies (31). During a merger, galaxies approach each other very closely, and tidal forces cause distortions in their shapes. In recent years, it has become possible to test the hypothesis of fueling via mergers by comparing the environments and shapes of galaxies with actively growing black holes to carefully chosen control samples of galaxies with dormant black holes. These studies have demonstrated that there is no clear connection between mergers and the fueling of black holes in the present-day universe $(32,33)$.

There is a strong connection observed between star formation in the central region of a galaxy
This radiation from these stars exerts pressure on the surrounding dusty medium, blowing apart the dense, cold cocoons of gas in which the stars were born. The stars rapidly burn through their supply of nuclear fuel and die in spectacular fashion in explosive events called supernovae, which violently heat the surrounding gas. When the rate at which massive stars are formed and die becomes large enough, the combined effect of all the supernova explosions powers an outflowing galactic wind (Fig. 4) that can sweep away the galaxy's gas and carry it out into the halo $(34,35)$. 


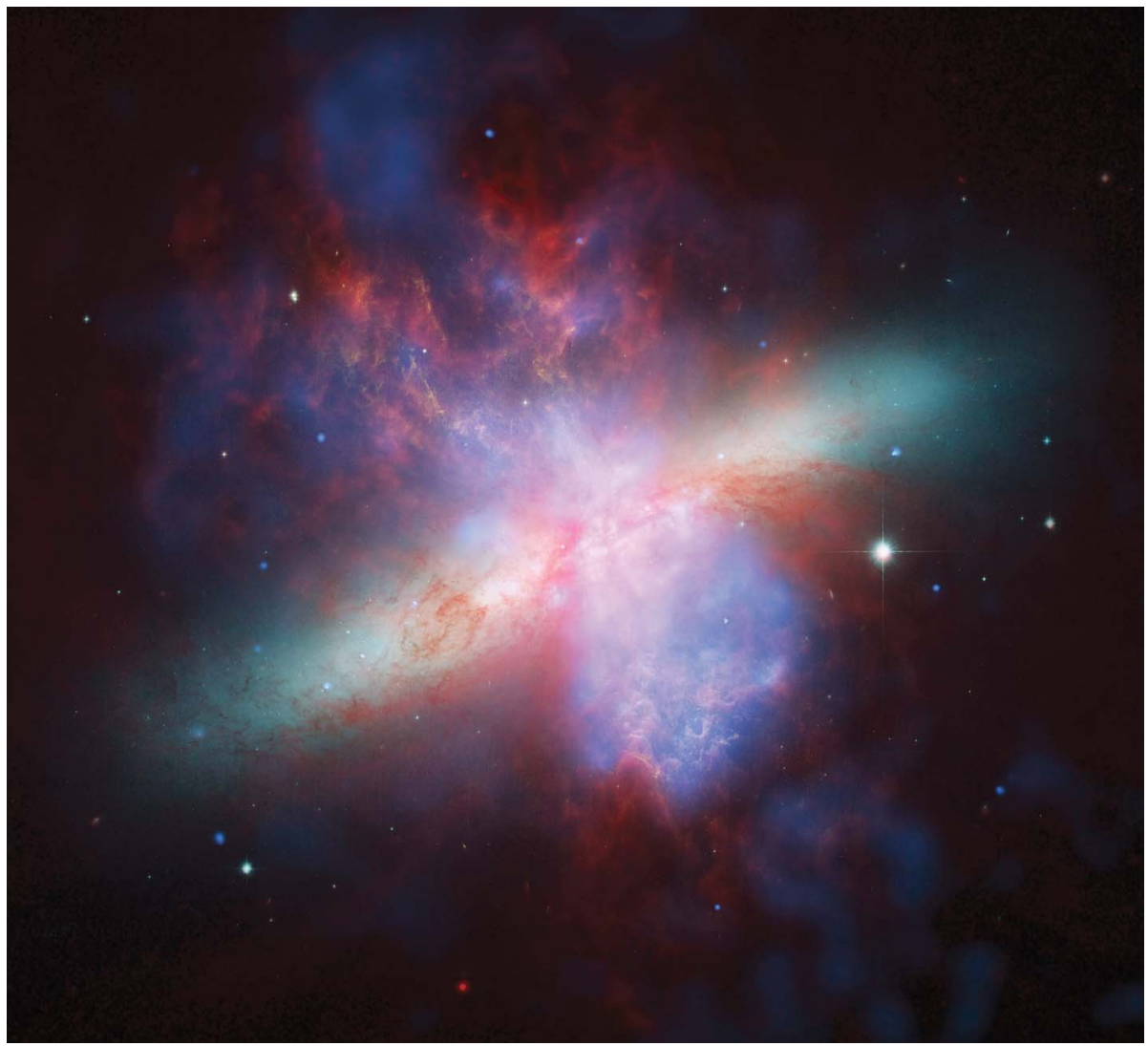

Fig. 4. Feedback driven by a burst of formation of massive stars in the galaxy Messier 82. This picture combines a true-color optical image taken by the Hubble Space Telescope of the galaxy itself (the aqua and pink band of light running from left to right across the image) with an image taken by the Chandra X-ray Observatory of a hot wind of gas traveling out into the halo (shown in blue) and with an infrared image taken by the Spitzer Space Telescope of much cooler gas and dust being carried in the outflow (shown in red). [Source: $x$-ray, NASA/CXC/]ohns Hopkins University/D. Strickland; optical, NASA/ESA/STScI/AURA/Hubble Heritage Team; infrared, NASA/]et Propulsion Laboratory-Caltech/University of Arizona/C. Engelbracht]

It is not yet clear whether this kind of feedback can really remove so much gas from the galaxy that the galaxy is transformed from living to passive, as is assumed in some theoretical models. It may be that gas is removed only from the central region of the galaxy, limiting the growth of the inner bulge and the black hole $(36,37)$. Could this process lead to the 1000 to-1 mass ratio?

Although star formation rates in these regions may decrease for some time, fresh gas that is brought in from the outer disk or ejected from dying stars may rejuvenate the galaxy once more, leading to a cycle of birth, death, and rebirth (38).

\section{Summary and Future Prospects}

Over the past decade, we have learned that not only do supermassive black holes exist, but that they are a crucial component of a complex cosmic ecosystem that links them together with dark matter, stars, and gas. This ecosystem has evolved dramatically over the history of the universe, and supermassive black holes have played a surprisingly major role in this evolution. Fortunately, we now have a working conceptual to come on several fronts. First, with the Atacan able to map out the detailed properties of the gas holes in present-day galaxies. This is the "for source" for the supermassive black hole, and we earn precisely how this food is delivered and the next generation of giant ground-based optical-infrared telescopes and x-ray observatories will work in concert with ALMA to provide detailed information about how star-formation, gas, and feedback processes are related to the formation and growth of supermassive black holes in the early universe. NASA's NuStar mission will be able to peer into the dust-enshrouded regions where many black holes are growing. Finally, there is an urgent need for large, complete surveys of the early universe that combine imaging with spectroscopy. This would give us a robust picture of the overall demographics of galaxies and supermassive black holes at early times. This could be compared to the demographics of the present-day universe, as has been determined by the Sloan Digital Sky Survey. Several such projects are under development.

It has been an exciting and surprising decade. The future looks even better.

\section{References and Notes}

1. L. Ferrarese, H. Ford, Space Sci. Rev. 116, 523 (2005).

2. R. Genzel, F. Eisenhauer, S. Gillessen, Rev. Mod. Phys. 82, 3121 (2010)

3. A. Ghez et al., Astrophys. J. 689, 1044 (2008).

4. L. Ho, Annu. Rev. Astron. Astrophys. 46, 475 (2008).

5. W. N. Brandt, G. Hasinger, Annu. Rev. Astron. Astrophys. 43, 827 (2005).

6. A. Marconi et al., Mon. Not. R. Astron. Soc. 351, 169 (2004).

7. I. Baldry et al., Astrophys. J. 600, 681 (2004).

8. D. Schiminovich et al., Astrophys. J. Suppl. Ser. 173, 315 (2007).

9. M. Brown et al., Astrophys. J. 654, 858 (2007)

10. S. Faber et al., Astrophys. J. 665, 265 (2007).

11. K. Gültekin et al., Astrophys. J. 698, 198 (2009).

12. A. Marconi, L. Hunt, Astrophys. J. 589, L21 (2003).

13. N. Häring, H.-W. Rix, Astrophys. J. 604, L89 (2004).

14. T. M. Heckman et al., Astrophys. J. 613, 109 (2004).

15. J. Greene, L. Ho, Astrophys. J. 667, 131 (2007).

16. L. Cowie et al., Astron. J. 112, 839 (1996).

17. K. Noeske et al., Astrophys. J. 660, L47 (2007).

18. B. Panter, R. Jimenez, A. F. Heavens, S. Charlot, Mon. Not. R. Astron. Soc. 378, 1550 (2007).

19. D. Croton et al., Mon. Not. R. Astron. Soc. 365, 11 (2006).

20. P. Hopkins et al., Astrophys. J. Suppl. Ser. 163, 1 (2006)

21. G. Kauffmann, M. Haehnelt, Mon. Not. R. Astron. Soc. 311, 576 (2000)

22. V. Springel, T. Di Matteo, L. Hernquist, Mon. Not. R. Astron. Soc. 361, 776 (2005).

23. A. Saintonge et al., Mon. Not. R. Astron. Soc., http://arxiv.org/abs/1103.1642 (2011).

24. B. R. McNamara, P. E. J. Nulsen, Annu. Rev. Astron. Astrophys. 45, 117 (2007).

25. P. Best, C. R. Kaiser, T. M. Heckman, G. Kauffmann, Mon. Not. R. Astron. Soc. 368, 167 (2006).

26. E. Churazov, M. Bruggen, C. R. Kaiser, H. Bohringer, W. Forman, Astrophys. J. 554, 261 (2001).

27. G. Kauffmann et al., Mon. Not. R. Astron. Soc. 346, 1055 (2003).

28. G. Kauffmann et al., Astrophys. J. Suppl. Ser. 173, 357 (2007)

29. G. Kauffmann, T. M. Heckman, Mon. Not. R. Astron. Soc. 397, 135 (2009)

30. H. Netzer, Mon. Not. R. Astron. Soc. 399, 1907 (2009).

31. D. B. Sanders, I. F. Mirabel, Annu. Rev. Astron. Astrophys. 34, 749 (1996).

32. C. Li, G. Kauffmann, T. M. Heckman, S. D. M. White, Y. P. Jing, Mon. Not. R. Astron. Soc. 385, 1915 (2008).

33. T. Reichard et al., Astrophys. J. 691, 1005 (2009).

34. T. M. Heckman, L. Armus, G. Miley, Astrophys. J. Suppl. Ser. 74, 833 (1990).

35. S. Veilleux, G. Cecil, ]. Bland-Hawthorn, Annu. Rev. Astron. Astrophys. 43, 769 (2005).

36. R. Davies et al., Astrophys. J. 671, 1388 (2007).

37. V. Wild, T. M. Heckman, S. Charlot, Mon. Not. R. Astron. Soc. 405, 933 (2010).

38. L. Ciotti, J. Ostriker, Astrophys. J. 665, 1038 (2007).

10.1126/science. 1200504 